\title{
On the Survival Assessment of Asthmatic Patients Using Parametric and Semi-Parametric Survival Models
}

\author{
Nureni Olawale Adeboye* ${ }^{*}$, Ilesanmi A. Ajibode, Olubisi L. Aako \\ Department of Mathematics \& Statistics, Federal Polytechnic, Ilaro, Ogun State, Nigeria \\ Email: *nureni.adeboye@federalpolyilaro.edu.ng, ilesanmi.ajibode@federalpolyilaro.edu.ng, \\ olubisi.aako@federalpolyilaro.edu.ng
}

How to cite this paper: Adeboye, N.O., Ajibode, I.A. and Aako, O.L. (2020) On the Survival Assessment of Asthmatic Patients Using Parametric and Semi-Parametric Survival Models. Occupational Diseases and Environmental Medicine, 8, 50-63. https://doi.org/10.4236/odem.2020.82004

Received: January 17, 2020

Accepted: April 11, 2020

Published: April 14, 2020

Copyright ( 2020 by author(s) and Scientific Research Publishing Inc. This work is licensed under the Creative Commons Attribution International License (CC BY 4.0).

http://creativecommons.org/licenses/by/4.0/ (c) (i) Open Access

\begin{abstract}
The goals of asthma management are to prevent or minimize symptoms, avert or reduce risk of asthma attacks and to ensure that asthma does not limit the patient's activities since it is not curable. Thus in this study, the degrees of success following treatments given to patients over time were assessed based on the patient's length of stay on admission and factors responsible for patients' response to treatment were equally examined using survival analysis models of parametric and semi-parametric distributions. The study was conducted on 464 asthmatic patients from four different hospitals in Ogun State. The data were extracted from patients' records and prognostic factors such as age, sex, smoking, hereditary, obesity, respiratory illness and environmental pollution were considered for survival analysis. It was observed that there was drastic reduction in survival rate from 7 days upward at a cut-off probability value of 0.485 , based on Kaplan-Meier (KM) results. Log-normal regression model, a parametric model with the least AIC value (2969.74) and least negative Log likelihood value (1475.87) shows best performance in handling asthma data with prognostic factors of Smoking ( $\mathrm{HR}=1.32,95 \%$ CI: $0.93-1.88$ ), Obesity ( $\mathrm{HR}=1.25,95 \% \mathrm{CI}: 0.80-1.93)$, Environmental pollution $(\mathrm{HR}=$ 0.79, 95\% CI: $0.52-1.18$ ) and Respiratory illness (HR $=1.93,95 \%$ CI: 1.33 2.79) were found to have significantly affected the length of stay of asthmatic patients in hospital.
\end{abstract}

\section{Keywords}

Asthma Disease, Cox Regression Model, Hazard Rates,

Parametric Regression Models, Prognostic Factors 


\section{Introduction}

Asthma has its origin from the Greek word, "aázein" meaning "to pant". Asthma can best be defined as a disease of the lung that causes narrowing of the airways resulting in the patient being unable to breathe properly. Asthma is a common and potentially serious chronic disease that imposes a substantial burden on patients, their families and the community. It causes respiratory symptoms, limitation of activity, and flare-ups (attacks) that sometimes require urgent health care and may be fatal. Also, the chronic inflammation is associated with airway hyper responsiveness that leads to recurrent episodes of wheezing, breathlessness, chest tightness and coughing particularly at night or early morning ref. [1]. Asthma is one of the world's commonest long-term conditions; the disease is estimated to affect as many as 300 million people worldwide and could increase by another 100 million by the year 2025. The World Health Survey (WHS) on asthma, championed by the World Health Organization (WHO) estimates the global burden of asthma in adults to be $4.3 \%$, with the highest burden in developed countries ref. [2]. Some of the various causes of asthma are genetic susceptibility and gene-environment interactions; environmental risk factors such as Prenatal factors, Indoor and outdoor allergens, Smoking and environmental tobacco smoke, other pollutants, Race/ethnicity, socioeconomic status, obesity, respiratory illnesses, etc.

The survival analysis has been used by many authors to study the survival of some ailments and factor responsible for their survival. The comparisons of parametric and semi-parametric model in survival analysis for describing and quantifying time to event have also been looked into in the literature. According to ref. [3], parametric models are only occasionally used in the analysis of clinical studies of survival although they may have advantages over Cox's model. Ref. [4] gave instances that researchers in medical sciences often tend to prefer Cox semi-parametric instead of parametric models for survival analysis because of fewer assumptions but under certain circumstances, parametric models give more precise estimates. Although Cox's semi-parametric model is the most frequently employed regression tool for survival data, fully parametric models may be better over some advantages. Based on asymptotic results, ref. [5] and ref. [6] opined that, under certain circumstances, parametric models lead to more efficient parameter estimates than Cox's model. With decreasing sample sizes, relative efficiencies may further change in favor of parametric models. When empirical information is sufficient, parametric models can provide some insight into the shape of the baseline hazard. Furthermore, extrapolations of survival functions become possible which though speculative may be of interest in some application.

Ref. [7] examined a non-response problem in survival analysis where the occurrence of missing data in the risk factor is related to mortality. The available data suggest that the process that created the missing data depends jointly on survival and the unknown blood pressure, thereby distorting the relation of interest. Ref. [8] carried out a survival analysis for patients with breast cancer. The 
influence of clinical and pathologic features, as well as molecular markers on survival time were investigated. Special attention focused on whether the molecular markers can provide additional information in helping predict clinical outcome and guide therapies for breast cancer patients. Ref. [9] examined Cox's regression model and parametric models in evaluating the prognostic factors for survival after liver transplantation in Shiraz during 2000-2012. Ref. [10] described the comparison of Cox and parametric regression models regarding survival of children with acute leukemia in southern Iran. Cox and parametric (exponential, Weibull, log-logistic, log-normal, Gompertz and generalized gamma) models were fitted to the data. The results showed that parametric regression models performed better compared to Cox model for identifying risk factors for prognosis with acute leukemia data. Ref. [11] compared cox model and parametric models in analysis of effective factors on event time of neuropathy in-patients with type 2 diabetes while ref. [12] compared Cox and Weibull Regression models in assessing the predictive factors for the survival of asthmatic patients treated at a federal medical center. It was shown that the parametric Weibull regression model could better determine the factors associated with asthma disease than the semi-parametric Cox-proportional hazard model.

There are several studies highlighting the burden of asthma among children in Nigeria, with a prevalence ranging from $5.1 \%$ to $14.3 \%$. Nevertheless, there remains a dearth of literature on the burden of asthma among adults in Nigeria ref. [13] [14]. The goals of asthma management are to prevent or minimize symptoms (control symptoms), avert or reduce risk of asthma attacks and to ensure that asthma does not limit the patient's activities since it is not curable. Thus in this study, the degree of success following treatments given to patients over time were assessed based on the patient's length of stay on admission. Factors responsible for patients' response to treatment are also examined using survival analysis models (parametric and semi-parametric) on Asthma disease data. The purpose of this study is to investigate the performance of Cox and parametric regression models in the survival analysis of the factors responsible for how long an asthmatic patient stays in hospital on admission. Attractiveness of Cox regression model to identify the prognostic factors especially in clinical research may be due to the model ability to deal with any distribution without any additional assumptions. However, it needs much more data to get reasonable results and does have the requirement of proportional hazards, which is not always satisfied by the data. If the assumption of proportional hazard does not hold, parametric survival models may perform better ref. [15]. Parametric models allow closed form expressions of the hazard function and other characteristics of the failure distribution.

\section{Methods}

\subsection{Data Sources and Method of Collection}

The target population for this study is asthma patients in selected federal and 
state hospitals in Ogun State, South western region of Nigeria. Ogun state is divided into four strata comprising Remo, Ijebu, Yewa and Egba. From each stratum, a major federal/state hospital is randomly selected based on availability. The selected hospitals are the Federal Medical Centre, Abeokuta, Ogun State Hospital, Ilaro, Ogun State Hospital, Remo and Ogun State Hospital, Ijebu Ode. The data for the research work were retrieved from the records of Asthmatic in-patients of the selected hospitals and it contains information on 464 patients who presented themselves for consultation on asthma related infections. The symptoms reported by the patients were recorded and information about the same patients were collected while undergoing treatment in the hospitals. Factors such as age, sex, heredity, smoking, obesity, environmental pollution, respiratory illness and patients' length of stay on admission were taken into consideration when collecting the data.

From the dataset, Ages of the patients are recorded in years and categorized as "2" for Adults (41 years and above); "1" for Young Adults (16 - 40 years) and "0" for Children ( 0 - 15 years) while gender were encoded in ordinal form as " 0 " for Female and "1" for Male. Other factors are encoded in integers ("0" for non-presence and " 1 " for the symptoms presence).

\subsection{Kaplan-Meier Estimator}

The Kaplan-Meier (KM) estimator is a very useful tool for estimating survival functions and the most popular in developing survival function. The KM estimator considers the probability of surviving an interval of time, given that a subject is at risk at the beginning of the interval. The Kaplan-Meier method can be used to estimate this curve from the observed survival times without the assumption of the underlying probability distribution ref. [16].

Let $t_{1}<t_{2}<\cdots<t_{D}$ denote the ordered event times in the sample. For $t_{i}$, let $d_{i}$ denote the total number of failures occurring at time $t_{i}, s_{i}$ denote the total number that haven't failed by time $t_{i}, n_{i}$ denote the total number at risk at time $t_{i}$, and $d_{i}=n_{i}-s_{i}$. Thus, ref. [17] estimate of survival time $S(t)$ is given by:

$$
\hat{S}_{K M}(t)=\prod_{i: t_{i} \leq t}\left(1-\frac{d_{i}}{n_{i}}\right)=\prod_{i: t_{i} \leq t}\left(\frac{s_{i}}{n_{i}}\right)
$$

and the Greenwood's formula for standard error estimate of the KM estimator is given by:

$$
\widehat{S E}_{G}\left\{\hat{S}_{K M}(t)\right\}=\hat{S}_{K M}(t) \sqrt{\sum_{i: t_{i} \leq t} \frac{d_{i}}{n_{i} S_{i}}}
$$

In the absence of censoring, the $\mathrm{KM}$ estimator reduces to the empirical survival function.

\subsection{Cox Proportional Hazard Model}

The proportional hazards model otherwise known as Cox regression model according to ref. [18] is a statistical technique for exploring the association be- 
tween the survival time of a patient and the several explanatory covariates. Cox method does not assume any particular distribution for survival times, rather it assumes that the effect of different explanatory variables on survival are constant over time and are additive in a particular way. The model assumes that the hazard for two individuals $x_{1}$ and $x_{2}$ is proportional.

The hazard is modeled as:

$$
h(t / x)=h_{o}(t) \exp \left(\beta_{1} x_{1}+\beta_{2} x_{2}+\cdots+\beta_{p} x_{p}\right)
$$

where $x_{1}, \cdots, x_{p}$ are explanatory variables. $h_{o}(t)=$ baseline hazard time $\mathrm{t}$ representing the hazard for a person with value 0 for all explanatory variables. $\beta_{1}, \cdots, \beta_{p}=$ regression coefficients describing the importance of the covariates, which is estimated by the partial likelihood estimation procedure.

Parameter estimates in Cox model are obtained by maximizing the partial likelihood function for the observed data simultaneously with respect to $h_{o}(t)$ and $\beta$ as proposed by ref. [19].

\subsection{Weibull Regression Model}

Weibull model is more general and flexible than exponential model and allow for hazard rates that are non-constant and proportional to a power of time $t$ but monotonic. $T$ is Weibull with parameters $\lambda$ and $p$, denotedas $T \sim W(\lambda, p)$. The survival function of the modelis $S(t)=\exp \left\{-(\lambda t)^{p}\right\}$, the hazard function is $h(t)=\lambda^{p} p t^{p-1}$ and the cumulative hazard is $\Lambda(t)=(\lambda t)^{p}$.

The log of the Weibull hazard is a linear function of log time with constant $p \log \lambda+\log p$ and slope $p-1$. Thus, the hazard is rising if $p>1$, constantif $p=1$, and declining if $p<1$.

The Weibull is also related to the extreme-value distribution: $T \sim W(\lambda, p)$ if and only if

$$
Y=\log T=\alpha+\sigma W
$$

where $W$ has the extreme value distribution, $\alpha=-\log \lambda$ and $p=\frac{1}{\sigma}$.

The survival function of $T$ at covariate value $z=\left(1, z_{1}, \cdots, z_{m}\right)^{T}$ can be shown to be

$$
S(t \mid z)=\exp \left\{-\left[t \mathrm{e}^{-\mathrm{z}^{\mathrm{T}} \beta}\right]^{\frac{1}{\lambda}}\right\}
$$

where $\beta=\left(\beta_{0}, \cdots, \beta_{m}\right)^{\mathrm{T}}$ is the vector of regression coefficients.

\subsection{Gompertz Model}

The Gompertz distribution is characterized by the fact that the log of thehazard is linear in $t$, so

$$
\lambda(t)=\exp (\alpha+\beta t)
$$

It is closely related to the Weibull distribution where the log of thehazard is linear in $\log t$. The hazard of Gompertz increases exponentially with $t$. In fact, 
the Gompertz is a log-Weibull distribution.

\subsection{Log-Normal}

$\mathrm{T}$ has a lognormal distribution if and only if

$$
Y=\log T=\alpha+\sigma W
$$

where $W$ has a standard normal distribution. The hazard function of the log-normal distribution increases from 0 to reach a maximum and then decreases monotonically, approaching 0 as tapproaches infinity. It is positively-skewed with density.

\subsection{Log-Logistic}

$T$ has a log-logistic distribution if and only if $W$ of log-normal has a standard logistic distribution, with pdf

$$
f_{W}(w)=\frac{\mathrm{e}^{w}}{\left(1+\mathrm{e}^{w}\right)^{2}}
$$

and cdf

$$
F_{W}(w)=\frac{\mathrm{e}^{w}}{1+\mathrm{e}^{w}}
$$

The survivor function is the complement

$$
S_{W}(w)=\frac{1}{1+\mathrm{e}^{w}}
$$

Changing variables to $T$ we find that the log-logistic survivor function is

$$
S(t)=\frac{1}{1+(\lambda t)^{p}}
$$

where $\alpha=-\log \lambda$ and $p=\frac{1}{\sigma}$. Taking logs the (negative) integrated hazard is obtained, and differentiating w.r.t. $t$ the hazard function

$$
\lambda(t)=\frac{\lambda p(\lambda t)^{p-1}}{1+(\lambda t)^{p}}
$$

A general approach to parametric families is to pick one of the distributions and let the parameters of the distribution depend on covariates.

\subsection{Model Selection Criteria}

The model selection criteria used in this research are Negative Log-likelihood and Akaike information criterion (AIC). AIC is a measure of the relative quality of statistical models for a given set of data. It estimates the quality of model, relative to each of the other models. It deals with the tradeoff between the goodness of fit of the model and the complexity of the model. It is defined as:

$$
\text { AIC }=-2\left(\frac{\ell}{T}\right)+\frac{2 K}{T}
$$


where $\ell=\log$ likelihood value, $K=$ number of parameters and $T=$ Total number of observations

The aim is to find the model with the lowest value of selected information criterion.

\section{Results}

The status of patients were classified as " 1 " for those that were discharged within forty (40) days of staying in the hospital while category zero (0) was used for patients that stay much more longer for treatments or whom eventually died as a results of the illness. The socio-demographic and clinical characteristics of the patients are as presented in Table 1 below.

Table 1. Socio-demographic and clinical characteristics analysis.

\begin{tabular}{|c|c|c|}
\hline Factors & Frequency & Percentage (\%) \\
\hline \multicolumn{3}{|l|}{ Gender } \\
\hline Male & 272 & 58.6 \\
\hline Female & 192 & 41.4 \\
\hline Total & 464 & 100.0 \\
\hline \multicolumn{3}{|l|}{ Age Group } \\
\hline Adults & 146 & 31.5 \\
\hline Young Adults & 185 & 39.9 \\
\hline Children & 133 & 28.7 \\
\hline Above 50 years & 33 & 24.3 \\
\hline Total & 464 & 100.0 \\
\hline \multicolumn{3}{|l|}{ Smoking } \\
\hline Yes & 83 & 17.9 \\
\hline No & 381 & 82.1 \\
\hline Total & 464 & 100.0 \\
\hline \multicolumn{3}{|l|}{ Hereditary } \\
\hline Yes & 182 & 39.2 \\
\hline No & 282 & 60.8 \\
\hline Total & 464 & 100 \\
\hline \multicolumn{3}{|l|}{ Obesity } \\
\hline Yes & 50 & 10.8 \\
\hline No & 414 & 89.2 \\
\hline Total & 464 & 100 \\
\hline \multicolumn{3}{|l|}{ Respiratory } \\
\hline Yes & 398 & 85.8 \\
\hline No & 66 & 14.2 \\
\hline Total & 464 & 100 \\
\hline \multicolumn{3}{|l|}{ Environmental } \\
\hline Yes & 55 & 11.9 \\
\hline No & 409 & 88.1 \\
\hline Total & 464 & 100 \\
\hline
\end{tabular}

Source: Field Survey, 2020. 
According to Table 1, 192 (41.4\%) were females while the remaining 272 (58.6\%) were males. 133 (28.7\%) were children, 185 (39.9\%) were young adults and $146(31.4 \%)$ were adults. 83 (17.9\%) were smokers and $381(82.1 \%)$ were non-smokers. $182(39.2 \%)$ inherited the disease, 50 (10.8\%) were obese, 398 $(85.8 \%)$ had respiratory illness and 55 (11.9\%) were affected by environmental pollution.

Kaplan-Meier survival analysis procedure was used to examine the distribution of length of stay of asthmatic patients in the hospital, while the appropriate model for survival analysis was fitted for the data as presented in the following results.

Table 2 and Figure 1 show that there is high survival rate within the first 7 days. The survival rate declined drastically after the seventh day. This is an indication that the probability of leaving the hospital after 7 days is slim. The chance of leaving hospital after 40 days is 0.18 .

Semi-parametric Cox regression and parametric regression models (Weibull, Log-logistic, Log-normal and Gompertz) were fitted to asthmatic patients' data in order to determine the best model. The model with lowest AIC and Negative log-likelihood will be adjudged the best model to describe the prognostic factors of survival of asthmatic patients in terms of their length of stay in hospital upon admission.

In Table 3, Cox and Gompertz models have negative coefficients for respiratory illness, age, sex, smoking, hereditary and obesity, and positive coefficient for environmental pollution. The reverse is the case for Log-logistic, Weibull and Log-normal models which have positive coefficients for respiratory illness, age,

Table 2. KM survival function.

\begin{tabular}{ccccccc}
\hline $\begin{array}{c}\text { Time } \\
\text { (days) }\end{array}$ & No. Risk & No. Event & Survival & $\begin{array}{c}\text { Std. } \\
\text { Error }\end{array}$ & $\begin{array}{c}\text { Lower } \\
95 \% \text { CI }\end{array}$ & $\begin{array}{c}\text { Upper } \\
95 \% \text { CI }\end{array}$ \\
\hline 1 & 464 & 27 & 0.942 & 0.0109 & 0.921 & 0.963 \\
2 & 437 & 40 & 0.856 & 0.0163 & 0.824 & 0.888 \\
3 & 397 & 43 & 0.763 & 0.0197 & 0.725 & 0.803 \\
4 & 354 & 30 & 0.698 & 0.0213 & 0.658 & 0.741 \\
5 & 324 & 21 & 0.653 & 0.0221 & 0.611 & 0.698 \\
6 & 303 & 19 & 0.612 & 0.0226 & 0.569 & 0.658 \\
7 & 284 & 59 & 0.485 & 0.0232 & 0.442 & 0.533 \\
10 & 214 & 20 & 0.442 & 0.0231 & 0.399 & 0.489 \\
15 & 175 & 31 & 0.375 & 0.0225 & 0.333 & 0.422 \\
20 & 167 & 5 & 0.364 & 0.0223 & 0.323 & 0.411 \\
25 & 125 & 42 & 0.272 & 0.0207 & 0.235 & 0.316 \\
30 & 116 & 11 & 0.248 & 0.0201 & 0.212 & 0.291 \\
35 & 86 & 29 & 0.185 & 0.0181 & 0.153 & 0.224 \\
40 & 81 & 4 & 0.177 & 0.0178 & 0.145 & 0.215 \\
\hline
\end{tabular}




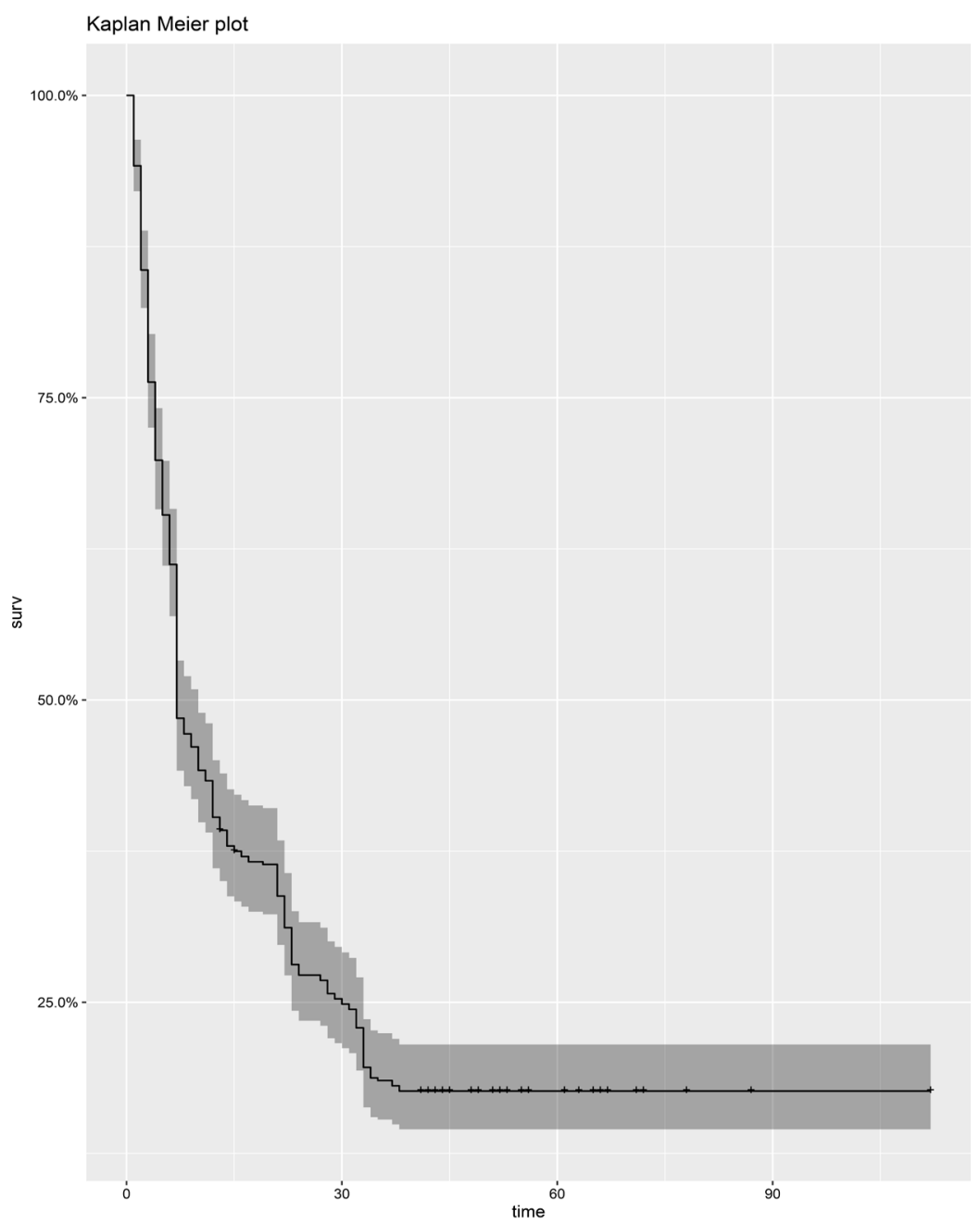

Figure 1. Kaplan-Meier's curve.

Table 3. Comparison of cox and parametric regression models.

\begin{tabular}{llllll}
\hline & Cox & Log-Logistic & Weibull & Gompertz & Log-Normal \\
\hline Shape & NA & 0.1860 & -0.2447 & -0.0444 & 1.5397 \\
Scale & NA & 1.4409 & 2.0103 & -1.7228 & 0.3316 \\
RES.ILLNESS & -0.58635 & 0.7036 & 0.7794 & -0.5627 & 0.6569 \\
AGE & -0.10079 & 0.1199 & 0.1203 & -0.0921 & 0.1325 \\
SEX & -0.09763 & 0.9307 & 0.1486 & -0.0982 & 0.0802 \\
SMOKING & -0.26361 & 0.3104 & 0.3758 & -0.2592 & 0.2770 \\
HERED. & -0.08828 & 0.1455 & 0.1470 & -0.0999 & 0.1320 \\
OBESITY & -0.17333 & 0.1407 & 0.3031 & -0.1650 & 0.2200 \\
ENV.POL. & 0.23267 & -0.2025 & -0.3263 & 0.2228 & -0.2419 \\
AIC & 4189.67 & 2980.68 & 3054.32 & 2973.69 & 2969.74 \\
NEG. LOGLIK & 2100.47 & 1481.30 & 1518.16 & 1477.84 & 1475.87
\end{tabular}


sex, smoking, hereditary and obesity, and negative coefficient for environmental pollution. A unit increase in variable with negative coefficient leads to decrease in length of stay in hospital while a unit increase in variable with positive coefficient leads to increase in length of stay in hospital. The AIC criterion and negative log-likelihood of Cox model were the highest $(4189.67,2100.47)$ and among the parametric models, Log-normal has the lowest AIC (2969.74) and negative log-likelihood (1475.87) follow by that of Gompertz model. Hence, Log-normal will best describe the prognostic factors for the survival of asthmatic patients.

Figure 2 presented the survival plot of the considered models with log-normal curve providing the best fit.

According to the result of Log-normal model analysis in Table 4, the hazard rate of length of stay of asthmatic patients due to respiratory illness increase by $93 \%(\mathrm{HR}=1.93,95 \% \mathrm{CI}: 1.33-2.79)$, male patients have hazard rate of $8 \%$ (HR $=1.08,95 \%$ CI: $0.83-1.41$ ) more than female in the reference category, adult (above 40 years) and young adult (16 - 40 years) have hazard rate of $14 \%(\mathrm{HR}=1.14$, 95\% CI: $0.94-1.39)$ more than children ( 0 - 15 years) in the reference category.

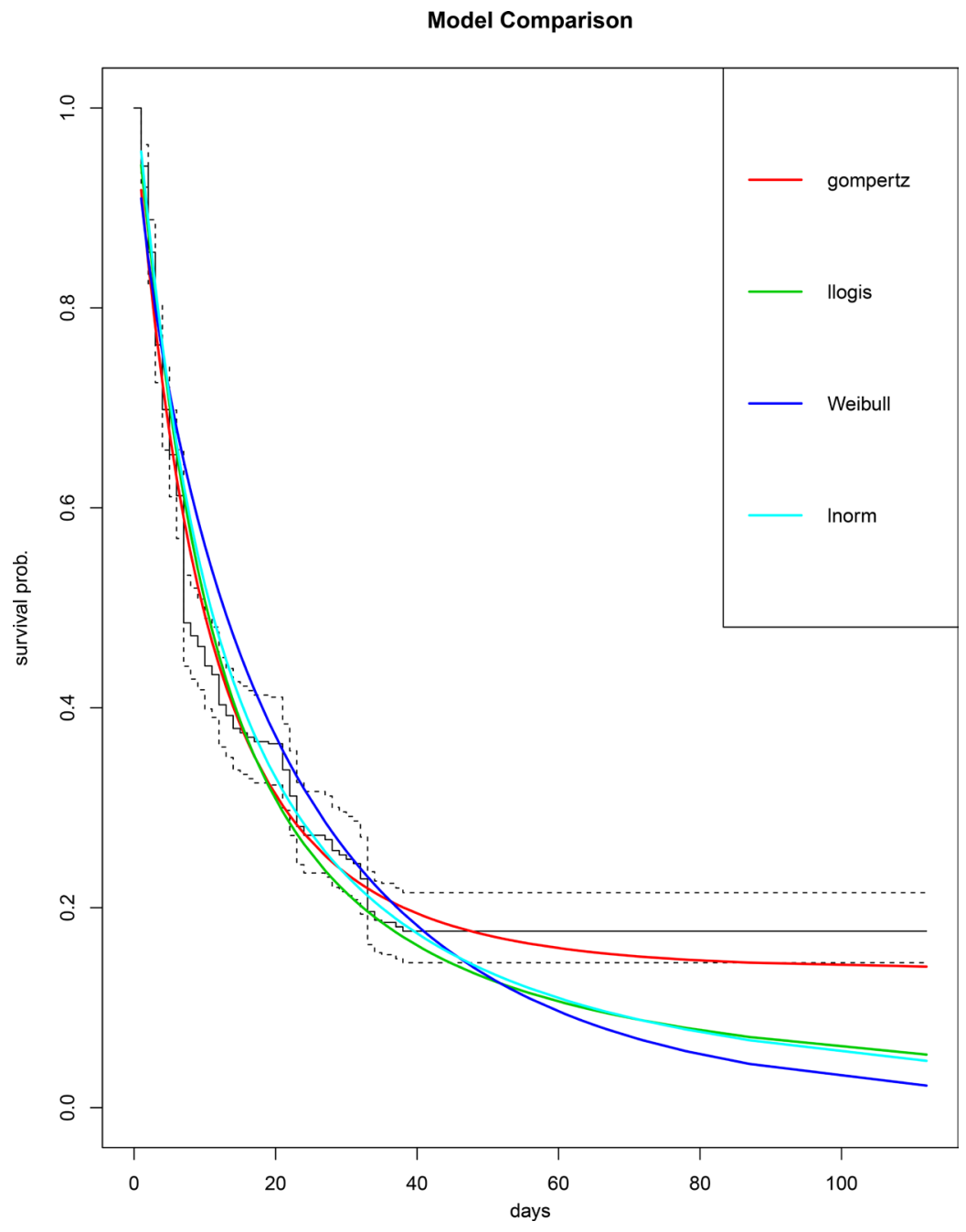

Figure 2. Survival fit of cox and parametric regression models. 
Table 4. Prognostic factors of asthmatic patients using log-normal model.

\begin{tabular}{cccccccc}
\hline & Coef. & $\begin{array}{c}\text { L95\% } \\
\text { C.I }\end{array}$ & $\begin{array}{c}\text { U95\% } \\
\text { C.I }\end{array}$ & $\begin{array}{c}\text { Std. } \\
\text { Error }\end{array}$ & $\begin{array}{c}\text { Hazard } \\
\text { Ratio(HR) }\end{array}$ & $\begin{array}{c}\text { L95\% } \\
\text { C.I }\end{array}$ & $\begin{array}{c}\text { U95\% } \\
\text { C.I }\end{array}$ \\
\hline Meanlog & 1.5397 & 1.0666 & 2.0127 & 0.2414 & NA & NA & NA \\
Sdlog & 1.3932 & 1.2932 & 1.5011 & 0.0530 & NA & NA & NA \\
RES.ILL. & 0.6569 & 0.2873 & 1.0264 & 0.1885 & 1.9287 & 1.3329 & 2.7911 \\
AGE & 0.1325 & -0.0644 & 0.3295 & 0.1005 & 1.1417 & 0.9376 & 1.3903 \\
SEX & 0.0802 & -0.1847 & 0.3452 & 0.1352 & 1.0835 & 0.8314 & 1.4122 \\
SMOKING & 0.2770 & -0.0767 & 0.6308 & 0.1805 & 1.3192 & 0.9262 & 1.8790 \\
HERED. & 0.1320 & -0.1891 & 0.4530 & 0.1638 & 1.1411 & 0.8277 & 1.5731 \\
OBESITY & 0.2197 & -0.2197 & 0.6590 & 0.2242 & 1.2457 & 0.8027 & 1.9329 \\
ENV.POL & -0.2419 & -0.6495 & 0.1657 & 0.2080 & 0.7851 & 0.5223 & 1.1803 \\
\hline
\end{tabular}

The hazard rate due to smoking increases by $32 \%$ (HR $=1.32,95 \%$ CI: 0.93 $1.88)$, hereditary factor increases the hazard rate by $14 \%(\mathrm{HR}=1.14,95 \% \mathrm{CI}$ : $0.83-1.57)$, hazard rate due to obesity increases by $25 \%(\mathrm{HR}=1.25,95 \% \mathrm{CI}$ : $0.80-1.93)$ and the hazard rate due to environmental pollution decreases by $21 \%(\mathrm{HR}=0.79,95 \%$ CI: $0.52-1.18)$.

\section{Discussion}

The results is the analysis of data attributed to 464 in-patients collected from the four selected hospitals, out of which 83 (17.9\%) were rightly censored at 40 days treatment periods. The median survival time was estimated to be at most 7 days with a cut-off probability of 0.485 , indicating that less than $48.5 \%$ of the asthmatic patients stayed less than 7 days and the other 51.5\% stayed longer. It indicates that $48.5 \%$ of the patients were discharged on or before the seventh day following success of treatments given to patients over time while the remaining $51.5 \%$ occupy bed spaces for more than 7 days or they were eventually discharged without surviving the illness. Overall survival (time in days) of asthmatic patients was calculated and represented graphically as presented in Figure 1, using KM curve with upper and lower estimates. Table 2 and Figure 1 show that there is high survival rate within the first 7 days. The survival rate declined drastically after the seventh day. This is an indication that the probability of leaving the hospital alive after 7 days is slim as observed in the results with the chance of leaving hospital alive after 40 days estimated at 0.18 .

Semi-parametric Cox regression and parametric regression models (Weibull, Log-logistic, Log-normal and Gompertz) were fitted to asthmatic patients' data in order to determine the best model, and the model with the lowest AIC and Negative log-likelihood adjudged the best model to describe the effect of the prognostic factors on survival of asthmatic patients in terms of their length of stay in hospital upon admission. The results presented in Table 3 and Figure 2 showed that Cox and Gompertz models have negative coefficients for respiratory 
illness, age, sex, smoking, hereditary and obesity, and positive coefficient for environmental pollution. The reverse is the case for Log-logistic, Weibull and Log-normal models which have positive coefficients for respiratory illness, age, sex, smoking, hereditary and obesity, and negative coefficient for environmental pollution. The estimated coefficients explained the magnitudes of unit impacts, each factors had on every asthma patients based on the fitted models. The AIC criterion and negative log-likelihood of Cox model were the highest (4189.67, 2100.47) and among the parametric models, Log-normal has the lowest AIC (2969.74) and negative log-likelihood (1475.87) follow by that of Gompertz model. Hence, Log-normal will best describe the prognostic factors for the survival of asthmatic patients.

According to the result of Log-normal model analysis in presented in Table 4, the hazard rate of length of stay of asthmatic patients due to respiratory illness increase by $93 \%(\mathrm{HR}=1.93,95 \% \mathrm{CI}: 1.33-2.79)$, male patients have hazard rate of $8 \%$ ( $\mathrm{HR}=1.08,95 \% \mathrm{CI}: 0.83-1.41)$ more than female in the reference category, adult (above 40 years) and young adult (16 - 40 years) have hazard rate of $14 \%(\mathrm{HR}=1.14,95 \% \mathrm{CI}: 0.94-1.39)$ more than children $(0-15$ years $)$ in the reference category. The hazard rate due to smoking increases by $32 \%(\mathrm{HR}=$ 1.32, 95\% CI: 0.93 - 1.88), hereditary factor increases the hazard rate by $14 \%$ (HR $=1.14,95 \%$ CI: $0.83-1.57)$, hazard rate due to obesity increases by $25 \%(\mathrm{HR}=$ 1.25, 95\% CI: 0.80 - 1.93) and the hazard rate due to environmental pollution decreases by $21 \%$ ( $\mathrm{HR}=0.79,95 \% \mathrm{CI}: 0.52-1.18)$. The environmental pollution hazard rate is an indication that the patients under coverage were not reported to have exposed to serious cases of environmental pollution.

\section{Conclusions}

The performance of semi-parametric Cox regression model in comparison with parametric models such as Weibull, Log-logistic, Log-normal and Gompertz regression models were critically examined. The study of the prognostic factors as they affect the length of stay of asthmatic patients in hospital equally considered in this research, is essential for the management of illness in terms of health-care costs, after-discharge home care and bed occupancy in hospitals at different levels. This study thus assessed, the degree of success following treatments given to patients over time based on asthmatic patient's length of stay on admission. It was discovered that only $48.5 \%$ responded to treatment within the first seven days of their admission. Factors responsible for patients' response to treatment were examined using the best fitted survival analysis model. That is, Log-normal regression model with the minimum AIC and Negative Log-likelihood values. It was shown that age, smoking, hereditary, obesity, respiratory illness and environmental pollution had a significant effect but sex had no significant effect on the survival of asthmatic patients. Smoking, obesity and respiratory illness had more effect than other factors. It is expected that this present work can be useful to health managers and asthmatic patients. 
The factors identified to have prolonged the patients length of stay in the hospital are smoking, obesity, environmental pollution and respiratory illness with the latter having the highest hazard rate. This implies that majority of the patients in the south western part of Nigeria are exposed to respiratory endagered challenges. Specifically, the following listed benefits are the highlights of this research:

- The data on asthma infection could be useful for government and health workers to make decisions that would reduce the risk of asthma infection among the populace.

- The data analysis reveals high significant impacts of prevalent factors such as Smoking, obesity and respiratory illness on asthma morbidity.

- The research provides a deeper understanding of the prevalence and prognosis of asthma infection.

- The results can be useful in asthma infection awareness, management and treatment.

- The chances of an asthmatic in-patient surviving the treatment may not exceed seven (7) days.

- The asthma data could be used as a baseline for comparison in future studies.

\section{Acknowledgements}

The authors wish to acknowledge the financial support of Tertiary Education Trust Fund (TETFund), Nigeria, in funding this research. We are also grateful to the management of Ogun state hospital, Ilaro, Nigeria; Ogun State Hospital, Ishagamu-Remo, Nigeria; Ogun State Hospital, Ijebu Ode, Nigeria and Federal Medical Centre, Abeokuta, Nigeria, for making the data available for the purpose of this research. Finally, we want to appreciate the management of Federal Polytechnic Ilaro, Nigeria, through which the funding was facilitated.

\section{Conflicts of Interest}

The authors declare no conflicts of interest regarding the publication of this paper.

\section{References}

[1] Bateman, E.D., Hurd, S.S., Barnes, P.J., Bousquet, J., Drazen, J.M., Fitz Gerald, M., Gibson, P., Olita, K., O’Byrne, P., Petersen, S.E., Pizzichini, E., Sullivan, S.D., Wenzel, S.E. and Zar, H.J. (2008) Global Strategy for Asthma Management and Prevention-Burden Report. Geneva: Global Initiative for Asthma, European Respiratory Journal, 31, 143-178. http://www.ginasthma.com

[2] Stanojevic, S., To, T., Moores, G., Gershon, A.S., Bateman, E.D., Cruz, A.A. and Boulet, L.P. (2012) Global Asthma Prevalence in Adults: Findings from the Cross-Sectional World Health Survey. BMC Public Health, 19, 204. https://doi.org/10.1186/1471-2458-12-204

[3] Nardi, A. and Schemper, M. (2003) Comparing Cox and Parametric Models in Clinical Studies. Statistics in Medicine, 22, 597-610. https://doi.org/10.1002/sim.1592 
[4] Mohamed, A.P., Ebrahim, H., Bijan, M.D., Azadi, S., Alireza, A. and Mohammed, R.Z. (2007) Comparing Cox Regression and Parametric Models for Survival of Patients with Gatric Carcinoma. Asian Pacific Journal of Cancer Prevention, 8, 412-416.

[5] Efron, B. (1977) The Efficiency of Cox's Likelihood Function for Censored Data. Journal of the American Statistical Association, 72, 557-565. https://doi.org/10.1080/01621459.1977.10480613

[6] Oakes, D. (1977) The Asymptotic Information in Censored Survival Data. Biometrika, 64, 441-448. https://doi.org/10.1093/biomet/64.3.441

[7] Van Buuren, S., Boshuizen, H.C. and Knock, D.L. (1999) Multiple Imputation of Missing Blood Pressure Covariates in Survival Analysis. Statistics in Medicine, 18, 681-694. https://doi.org/10.1002/(SICI)1097-0258(19990330)18:6<681::AID-SIM71>3.0.CO;2-R

[8] Liu, Y. (2010) Survival Analysis for Breast Cancer. A Thesis Submitted in the Department of Mathematics and Statistics, University of Victoria, Victoria.

[9] Adelian, R., Jamali, J., Zare, N., Ayatollahi, S.M.T., Pooladfar, G.R. and Ronstaei, N. (2015) Comparison of Cox's Regression Model and Parametric Models in Evaluating the Prognostic Factors for Survival after Liver Transplantation in Shiraz during 2000-2012. The International Journal of Organ Transplantation Medicine, 6, 119-125.

[10] Teshnizi, S.H. and Ayatollahi, S.M.T. (2017) Comparison of Cox Regression and Parametric Models. Application for Assessment of Survival of Pediatric Cases of Acute Leukemia in Southern Iran. Asian Pacific Journal of Cancer Prevention, 18, 981-985.

[11] Sadegh, T. and Iraj, D. (2017) Comparison of Cox Model and Parametric Models in Analysis of Effective Factors on Event Time of Neuropathy in Patients with Type 2 Diabetes. Journal of Research in Medical Sciences, 22, 115. https://doi.org/10.4103/jrms.JRMS_6_17

[12] Ezekiel, I.D. and Aako, O.L. (2018) Comparison of Cox's and Weibull Regression Models in Assessing the Prognostic Factors for Survival of Asthmatic Patients. International Journal of Current Innovation Research, 4, 1390-1394.

[13] Musa, B.M. and Aliyu, M.D. (2014) Asthma Prevalence in Nigerian Adolescents and Adults: Systematic Review and Meta-Analysis. African Journal of Respiratory Medicine, 10, 4-9.

[14] Berwick, V., Cheek, L. and Bell, T. (2004) Statistical Review 12: Survival Analysis Crit. Core, 8, 389-394. https://doi.org/10.1186/cc2955

[15] Gardiner, J.C. (2010) Survival Analysis: Overview of Parametric, Nonparametric and Semi-Parametric Approaches and New Developments. Stat Data Anal Paper, 252.

[16] Gokovali, U., Bahar, O. and Kozak, M. (2007) Determinant of Length of Stay: A Practical Use of Survival Analysis. Tourism Management, 28, 736-746. https://doi.org/10.1016/j.tourman.2006.05.004

[17] Kaplan, E.L. and Meier, P. (1958) Non Parametric Estimation from Incomplete Observations. Journal of the American Statistical Association, 53, 457-481. https://doi.org/10.1080/01621459.1958.10501452

[18] Cox, D.R. (1972) Regression Models and Life-Tables (with Discussion). Journal of the Royal Statistical Society: Series B, 34, 187-202.

https://doi.org/10.1111/j.2517-6161.1972.tb00899.x

[19] Cox, D.R. and Oakes, D. (1984) Analysis of Survival Data. Chapman \& Hall, London. 